\title{
HIV/AIDS-Related Knowledge and Behaviors Among Most-at-Risk Populations in Vietnam
}

\author{
Taryn Vian ${ }^{* 1,2}$, Katherine Semrau ${ }^{1,2}$, Davidson H. Hamer ${ }^{1,2,3}$, Le Thi Thanh Loan ${ }^{4}$ and Lora L. Sabin ${ }^{1,2}$ \\ ${ }^{1}$ Center for Global Health and Development, Boston University, Boston, Massachusetts, USA \\ ${ }^{2}$ Department of International Health, Boston University School of Public Health, Boston, Massachusetts, USA \\ ${ }^{3}$ Infectious Diseases Section, Department of Medicine, Boston University School of Medicine, Boston, Massachusetts, \\ USA \\ ${ }^{4}$ Ho Chi Minh City Statistical Office, Ho Chi Minh City, Vietnam
}

\begin{abstract}
The U.S. President's Emergency Plan for AIDS Relief (PEPFAR) has supported the Vietnamese Ministry of Health $(\mathrm{MOH})$ in implementing behavior change strategies to slow the HIV epidemic. These programs target commercial sex workers (CSW), injection drug users (IDU), and men who have sex with men (MSM). Using data from a program evaluation to assess effectiveness of the PEPFAR intervention, we conducted a sub-analysis of HIV/AIDS knowledge, sexual behaviors, and injection drug risk behaviors among 2,199 Vietnamese respondents, including those reporting recent contact with an outreach worker and those who did not report contact. We found overall high levels of HIV/AIDS knowledge, low rates of needle sharing, and moderate to high rates of inconsistent condom use. Average knowledge scores of IDU were significantly higher than non-IDU for antiretroviral treatment knowledge, while MSM had significantly less knowledge of treatment compared to non-MSM. HIV/AIDS-related knowledge was not significantly associated with needle-sharing practices. Knowledge was modestly but significantly associated with more consistent use of condoms with primary and commercial sex partners, even after controlling for contact with an outreach worker. Contact with an outreach worker was also an independent predictor of more consistent condom use. Outreach programs appear to play a meaningful role in changing sexual behavior, though the effect of outreach on IDU risk behaviors was less clear. More research is needed to understand the relationship between outreach programs and skill development, motivation, and use of referral services by most-at-risk populations in Vietnam.
\end{abstract}

Keywords: HIV/AIDS, sex behavior, health communications, Vietnam, injection drug users, commercial sex workers, men who have sex with men, most-at-risk populations.

\section{INTRODUCTION}

HIV transmission in Vietnam is concentrated in most-atrisk populations (MARP), including commercial sex workers (CSW), injection drug users (IDU), and men who have sex with men (MSM) $[1,2]$. Prevalence in IDU is estimated at $34 \%$ [3], among CSW prevalence is approximately $16 \%$ nationally with higher rates in cities such as Hanoi and Ho Chi Minh City (HCMC) [3-5], and researchers estimate seroprevalence among MSM at 5.8\% [6]. IDU account for more than $65 \%$ of HIV infections in Vietnam [7] while CSW comprise 4\% [2]. In urban areas MSM represent $5-33 \%$ of total AIDS cases [8,9]. Reducing risky behaviors among MARP is therefore a priority in controlling HIV in Vietnam.

MARP in Vietnam continue to engage in behaviors which increase their risk of infection. According to one report, $12-33 \%$ of IDU share injection equipment [10]. In addition, condom use is uncommon among IDU and

*Address correspondence to this author at the Department of International Health, Boston University School of Public Health, 801 Massachusetts Avenue, Crosstown Building, Room 375, Boston, MA 02118, USA; Tel: 617-414-1447; Fax: 617 638-4476; E-mail: tvian@bu.edu inconsistent among CSW and MSM [1, 6, 11]. Overlap among the MARP groups heightens risk: many sex workers also inject drugs, including $20 \%$ of street-based sex workers in HCMC and $43 \%$ in Hanoi [10].

Community-based peer education interventions have proven effective in increasing knowledge and changing behavior, especially among high risk populations [12-14]. For example, researchers working with female partners of IDU in South Asia found that people who had been approached with HIV information were twice as likely to have used condoms during the last sexual intercourse, compared to people who had not been approached [15]. Programs providing information and service referral are associated with reduced drug use, reduced reuse of injection equipment, increased condom use, and increased uptake of services such as drug cessation treatment and voluntary counseling and testing (VCT) [16].

The U.S. President's Emergency Plan for AIDS Relief (PEPFAR) has supported the Vietnamese Ministry of Health $(\mathrm{MOH})$ in implementing behavior change communication programs. These programs employ peer educators and health educators to establish rapport with clients and provide information and educational messages, develop skills such as 
correct use of condoms and needle cleaning skills, and refer to services such as voluntary counselling and testing, HIV treatment, and drug use cessation treatment. Most peer education programs also provide condoms and bleach packets. Peer educators are often current or former IDU, CSW, or MSM, while health educators are usually university graduates with health education training. They undergo initial and refresher training programs, and are usually paid a small stipend. Individual programs are managed by different agencies or groups of agencies, including international and local non-governmental organizations and provincial health services or AIDS committees. These organizations set expectations for productivity of peer educators and health educators, including targets for client contacts per week.

From 2006-08, PEPFAR and the Government of Vietnam supported an evaluation of community outreach HIV prevention programs. The goal was to assess effectiveness in increasing knowledge and use of services by MARP, and identify lessons learned. The evaluation found that outreach workers were successful in making contact with MARP clients despite numerous challenges, and that the program had contributed to reduced risk behavior [17].

The purpose of this paper is to conduct a sub-analysis of the evaluation's findings regarding HIV/AIDS knowledge and behaviors of MARP. While the previous report described knowledge and behaviors, it did not try to relate them through regression analysis. Our hypothesis, which was one of the theoretical underpinnings of the community outreach intervention, was that greater knowledge would be associated with fewer risky behaviors.

\section{METHOD}

\section{Procedures}

The data analyzed came from a cross-sectional survey of MARP in four provinces (Hanoi, Hai Phong, HCMC, and An Giang). Surveys were translated using modified direct translation method, which allows for changes in order and grammar to obtain meaningful cultural equivalency [18]. Interviews were done face-to-face in locations agreed upon by the respondents, with privacy assured. Interviewers administered the questionnaire in Vietnamese. For further details see study report [17]. Data were collected between November 2007 and January 2008. The institutional review boards of Boston University Medical Center, the Hanoi School of Public Health, and the HCMC AIDS Committee approved the study.

\section{Sample}

Potential respondents were identified through respondent-driven sampling $[19,20]$ in which MARP participants were asked to convey information about the evaluation to MARP acquaintances, along with times when interviewers would be waiting to meet respondents.

\section{Data and Measures}

The survey instrument included questions on demographic information, knowledge on transmission, prevention and treatment, and HIV risk behaviors. Questions were based on questionnaires used previously in HIV research in Vietnam [10] and incorporated input from PEPFAR and the HCMC Statistical Office based on content of outreach education activities. Although the study predates the development of the national Monitoring and Evaluation framework with knowledge and behaviour indicators designed by the Vietnam Administration of HIV/AIDS Control (VACC), the study's indicators capture similar constructs, i.e. knowledge of correct ways to prevent transmission and rejection of major misconceptions, consistent use of condoms, and needle sharing behavior. For this analysis, the sample size was limited to those who answered the questions on HIV knowledge $(n=2,199 / 2,222$, or $98.9 \%)$.

Demographic variables collected included geographic location, age, gender, education, main daily activity, and HIV-related characteristics such as testing experience and results. To assess knowledge related to HIV transmission and possible misconceptions, we asked 16 yes/no questions such as "Can you become infected with HIV by having unprotected vaginal sex?" and "Can you become infected by hugging a person infected with HIV?" For HIV prevention knowledge, we asked 17 yes/no questions such as "Can you avoid or reduce the chance of becoming infected with HIV by using condoms when having vaginal sex?" For treatment knowledge, 8 questions used a true/false format, such as "ART (antiretroviral therapy) improves immune function." Participants received 1 point for a correct answer and no points for missing or incorrect answers. A total score was calculated for each category and converted into a 100 point band score by category and overall.

Regarding IDU risk behavior, we asked about sharing of needles during the last six months and the last time injecting. For sexual behavior, following methods used in previous studies [21], we included questions related to consistency of condom use (e.g. "How often do you use a condom?") during sexual intercourse in the last six months with three groups of partners: primary, casual, and commercial. Inconsistent use was defined as less than "always". As there was no accepted universal standard measure at time we did the research, we used most conservative measure, i.e. anything less than $100 \%$ condom use was considered inconsistent [22].

When comparing knowledge by risk group, we defined participants by reported behavior in the last six months: IDU were defined as having reported injecting drug use $(n=694)$, CSW were defined as having reported accepting drugs, goods, or money from a client in exchange for sex $(\mathrm{n}=1,367)$, and MSM were males who reported having had sex with someone of the same sex $(n=337)$. Individuals could be counted in more than one risk group if they reported engaging in multiple risk behaviors, which explains why the total count by risk group classification $(n=2,398)$ is greater than the overall sample analyzed $(n=2,199)$. Each risk group's knowledge was compared to knowledge of all others not in that risk group.

\section{Statistical Analysis}

We tested for statistical differences between groups and conducted bivariate logistic regression followed by multivariate logistic regression to adjust for potential confounders. Our dependent variables were sharing needles and inconsistent condom use in the last six months, while the independent variable of interest was knowledge. In the 
multivariate regression analysis, backward selection was used to assess the impact of various potential confounders. The full model included the following variables: knowledge, MARP group, age, province, education, employment, HIV status, and contact with an outreach worker ("contact"). Confounders that did not create more than $10 \%$ change in the odds ratio of the main relationship (knowledge and behavior) were excluded from the model. In the final adjusted models, we controlled for contact, location (one of the four provinces where the survey was conducted, with Hanoi as the reference location), and age (a continuous variable in years). A total knowledge score was calculated for each category. These were converted into the percent correct by category and overall.

\section{RESULTS}

We analyzed data from 2,199 adult MARP (Table 1). The sample included 1,100 who reported contact with an outreach worker in the past 6 months ("contact group") and 1,099 who reported no such contact ("no contact group"). This measure of exposure to peer education does not take into account the length, frequency, and depth of contact, which may be influential variables. We return to this point in the discussion.

There were few differences between participants in the contact group and no contact group except that contact group MARP were slightly older on average (29.6 vs 28.5 years, $\mathrm{p}<0.001$ ), and more likely to report having tested positive for HIV $(21.8 \%$ vs $15.5 \%, \mathrm{p}<0.01)$.

Overall knowledge was high in both contact and no contact groups, especially regarding modes of transmission and prevention strategies (Table 2). However, treatment knowledge was low. Over $25 \%$ of MARP were unaware that there is no cure for HIV/AIDS (data not shown).

The survey revealed specific knowledge gaps related to HIV transmission and prevention (see supplementary online table). Less than $75 \%$ of respondents knew that one could not get HIV/AIDS through mosquito bites; similarly, less than $72 \%$ knew that HIV could be transmitted through breastfeeding. Only $35 \%$ of respondents knew that practicing withdrawal during sex was an ineffective prevention method, and only $71 \%$ correctly identified "not kissing" as ineffective in preventing infection. Among no contact group respondents, $15 \%$ did not know that unprotected anal sex could transmit HIV versus $8 \%$ in the contact group.

Average knowledge scores of IDU were significantly higher than non-IDU for treatment: IDU scored $46.7 \%$ on treatment knowledge, compared to $39.4 \%$ for non-IDU $(p<0.001)$ (Table 3). CSW scored significantly lower than non-CSW on combined transmission and prevention knowledge, although the absolute differences were small ( $82.9 \%$ for CSW compared to $84.3 \%$ for non-CSW, $\mathrm{p}<0.01$ ). MSM had significantly less knowledge of treatment than non-MSM (33.0\% vs $43.0 \%$ for non-MSM, $\mathrm{p}<0.001)$.

Respondents reported less risky behavior related to injection drug use, compared to sexual behaviors and the use of condoms. For example, while only $14.1 \%$ of respondents who used injection drugs in the last six months reported sharing needles or syringes, many more MARP admitted inconsistent condom use with commercial clients (36.2\%), primary partners $(70.6 \%)$, and casual partners $(84.0 \%)$ (data not shown). Risky behaviors were significantly associated with lower levels of HIV/AIDS-related knowledge (Table 4).

Table 1. Demographics of 2,199 Vietnamese MARP

\begin{tabular}{|c|c|c|}
\hline Characteristic & $\mathbf{N}$ & $\%$ \\
\hline \multicolumn{3}{|l|}{ Location } \\
\hline Ha Noi & 808 & $36.7 \%$ \\
\hline Ho Chi Minh City (HCMC) & 785 & $35.7 \%$ \\
\hline Hai Phong & 306 & $13.9 \%$ \\
\hline An Giang & 300 & $13.6 \%$ \\
\hline Age in Years Mean (SD) & 29.0 & $(7.90)$ \\
\hline \multicolumn{3}{|l|}{ Gender $^{1}$} \\
\hline Male & 866 & $39.4 \%$ \\
\hline Female & 1275 & $58.0 \%$ \\
\hline Other & 57 & $2.6 \%$ \\
\hline \multicolumn{3}{|l|}{ Education } \\
\hline Primary or none & 576 & $26.2 \%$ \\
\hline Secondary (grade 6-9) & 906 & $41.2 \%$ \\
\hline High School or higher & 717 & $32.6 \%$ \\
\hline \multicolumn{3}{|l|}{ Main Daily Activity } \\
\hline Construction/farming/petty job & 446 & $20.3 \%$ \\
\hline Salaried job & 315 & $14.3 \%$ \\
\hline Sex worker & 746 & $33.9 \%$ \\
\hline Entertainment worker & 194 & $8.8 \%$ \\
\hline Student, housework, other & 287 & $13.1 \%$ \\
\hline Unemployed & 210 & $9.6 \%$ \\
\hline \multicolumn{3}{|l|}{ Intervention } \\
\hline Contact & 1100 & $50.0 \%$ \\
\hline \multicolumn{3}{|l|}{ HIV Related Characteristics } \\
\hline Ever been tested for HIV & 1354 & $61.6 \%$ \\
\hline \multicolumn{3}{|l|}{ Of Those Tested for HIV } \\
\hline Post-test counselled & 990 & $73.1 \%$ \\
\hline Received results & 1216 & $89.8 \%$ \\
\hline HIV + Results & 236 & $19.4 \%$ \\
\hline
\end{tabular}

MARP $=$ most-at-risk population; $\mathrm{SD}=$ standard deviation

${ }^{1}$ Gender was self-defined. The category "Other" included respondents who identified as neither male nor female, i.e. gay, trans, queer, bisexual. One missing is not shown for Gender, and one missing is not shown for Main Daily Activity.

Respondents who shared injection equipment in the last six months had lower knowledge scores than those who reported not sharing equipment $(73.9 \%$ vs $76.8 \%, \mathrm{p}=0.05)$, and respondents who reported inconsistent use of condoms with commercial clients scored lower on the knowledge test compared to respondents who used condoms consistently $(72.2 \%$ vs $76.1 \%, \mathrm{p}<0.001)$. Here too, though, the absolute differences were small. 
Table 2. Mean Knowledge Scores for Respondents who had Contact with Outreach Workers versus Respondents who had No Contact

\begin{tabular}{|c|c|c|c|c|}
\hline \multirow{2}{*}{ Knowledge of: } & All (n=2,199) & Contact Group (n=1,100) & No Contact Group (n=1,099) & Mean \% (SD) \\
\cline { 2 - 5 } & Mean \% (SD) & Mean \% (SD) & $86.1(13.8)$ & $<0.001$ \\
\hline \hline Transmission & $88.6(12.2)$ & $91.0(9.6)$ & $77.0(15.2)$ & $<0.001$ \\
\hline Prevention & $78.6(14.0)$ & $80.2(12.5)$ & $35.2(25.0)$ & $81.4(12.8)$ \\
\hline Treatment & $41.7(27.3)$ & $48.2(27.9)$ & $<0.001$ \\
\hline Transmission and prevention combined & $83.4(11.4)$ & $85.4(9.3)$ & $72.4(12.4)$ & $<0.001$ \\
\hline All knowledge & $75.3(11.6)$ & $78.2(10.0)$ & $<.001$ \\
\hline
\end{tabular}

$\mathrm{SD}=$ standard deviation.

Table 3. Knowledge by Specific Risk Group compared to All Other MARP

\begin{tabular}{|c|c|c|c|c|c|}
\hline Knowledge of: & Mean (\%) & SD & Mean (\%) & SD & p-Value \\
\hline & \multicolumn{2}{|c|}{ IDU $(n=694)$} & \multicolumn{2}{|c|}{ All Others $(n=1,504)$} & \\
\hline Treatment & 46.7 & 29.4 & 39.4 & 25.9 & $<0.001$ \\
\hline All knowledge & 76.4 & 12.1 & 74.8 & 11.4 & $<0.003$ \\
\hline Transmission and prevention & 82.9 & 12.0 & 84.3 & 10.2 & 0.002 \\
\hline Treatment & 41.0 & 26.8 & 42.9 & 28.0 & 0.10 \\
\hline \multirow[t]{2}{*}{ All knowledge } & 74.7 & 12.0 & 76.3 & 10.9 & 0.002 \\
\hline & \multicolumn{2}{|c|}{ Male MSM (n=337) } & \multicolumn{2}{|c|}{ All Others $(\mathrm{n}=1,862)$} & \\
\hline
\end{tabular}

$\mathrm{MARP}=$ most-at-risk population, $\mathrm{SD}=$ standard deviation, $\mathrm{MSM}=$ men who have sex with men, $\mathrm{IDU}=$ injection drug user, $\mathrm{CSW}=$ commercial sex worker.

Table 4. Mean Total Knowledge Scores by Risk Behavior Status

\begin{tabular}{|c|c|c|c|c|c|c|c|}
\hline Risk Behavior & \multicolumn{3}{|c|}{ YES } & \multicolumn{3}{|c|}{ NO } & \\
\hline Sexual Risk Behaviors $^{1}$ & $\mathrm{~N}$ & Mean & SD & $\mathrm{N}$ & Mean & SD & p-value \\
\hline Inconsistent condom use with commercial clients ${ }^{2}$ & 495 & 72.2 & 13.3 & 872 & 76.1 & 10.9 & $<0.001$ \\
\hline Inconsistent condom use with casual partner & 1662 & 74.9 & 11.9 & 316 & 76.9 & 9.7 & 0.004 \\
\hline Inconsistent condom use with primary partner & 988 & 75.2 & 11.5 & 411 & 77.1 & 10.4 & 0.003 \\
\hline Needle Sharing Behaviors & $\mathrm{N}$ & Mean & SD & $\mathrm{N}$ & Mean & SD & p-value \\
\hline Shared injection equipment in last six months & 98 & 73.9 & 13.8 & 594 & 76.8 & 11.8 & 0.05 \\
\hline Shared injection equipment last time injecting & 33 & 73.1 & 13.6 & 658 & 76.6 & 0.5 & 0.10 \\
\hline
\end{tabular}

$\mathrm{SD}=$ standard deviation

${ }^{1}$ In last six months. YES indicates those who engaged in the behaviour, NO indicates those who did not.

${ }^{2}$ Denominator is those who reported receiving cash, drugs, or goods from a client for sex.

\section{Multivariable Regression Analyses}

We first looked at the unadjusted associations between variables of interest (knowledge, location, age, and contact) and IDU needle sharing (Table 5).
We found that age and location were significantly associated with needle sharing. Older persons were less likely to share needles, while residents of HCMC were three times more likely to share needles compared to Hanoi residents. There was a weak though statistically significant negative association between knowledge and needle sharing. 
In the adjusted multivariate analysis, location and age remained significant predictors of needle sharing but knowledge was no longer significant. We included the variable of contact with an outreach worker in the model with the expectation that it would be significantly related to risk behavior in the unadjusted model, but not in the multivariate analysis as the effect of contact would be mediated by knowledge. We found that contact was not significant in predicting needle sharing behavior in either the unadjusted or adjusted model.

Table 5. Multivariable Logistic Regression Analysis of the Effect of HIV-Related Knowledge on Needle Sharing Behavior in Last Six Months, Unadjusted and Adjusted (n=692)

\begin{tabular}{|c|c|c|}
\hline Outcome & \multicolumn{2}{|c|}{ Shared Needles (n=692) } \\
\hline Exposures & Unadjusted & Adjusted* \\
\hline \hline All Knowledge, \% & $0.98(0.97,0.99)$ & $0.99(0.97,1.01)$ \\
\hline Location & & \\
\hline An Giang & $1.08(0.51,2.28)$ & $0.71(0.33,1.55)$ \\
\hline HCMC & $3.03(1.82,5.03)$ & $2.45(1.44,4.16)$ \\
\hline Hai Phong & $0.57(0.24,1.36)$ & $0.75(0.31,1.80)$ \\
\hline Hanoi & reference & reference \\
\hline Age & $0.92(0.88,0.95)$ & $0.93(0.89,0.96)$ \\
\hline Contact $v s$ No Contact & $0.98(0.63,1.50)$ & $1.15(0.72,1.83)$ \\
\hline
\end{tabular} $\begin{aligned} & \text { Each item adjusted for all other variables in table. Confidence interval is indicated in } \\
& \text { parentheses. } \\
& \text { HCMC = Ho Chi Minh City. }\end{aligned}$

Next, we analyzed the unadjusted and adjusted associations between the same independent variables and inconsistent condom use (Table 6).

With primary partners, knowledge and contact were significant predictors of condom use in both models. In the adjusted model, location was also significant. An Giang residents were more likely to be consistent condom users, and contact reduced inconsistent condom use by nearly $30 \%$.
With casual partners, both knowledge and contact were significantly associated with inconsistent condom use in the unadjusted model. In addition, MARP in An Giang and Hai Phong were more likely to use condoms inconsistently compared to those in Hanoi. After adjustment, knowledge and contact were no longer significant although the other relationships held.

Finally, focusing on commercial clients, knowledge, contact, age, and location were all significantly associated with condom use in the unadjusted model. After adjustment, age was no longer significant and the association of knowledge with condom use was weak although significant. Location was still significant; people living in Hai Phong were more likely to use condoms consistently with commercial clients. Respondents who had contact with outreach workers were $32 \%$ more likely to report always using condoms.

\section{DISCUSSION}

In this analysis of knowledge and behaviors among MARP in Vietnam, we found generally high levels of HIV/AIDS knowledge, low rates of needle sharing, and moderate to high rates of inconsistent condom use. For IDUrelated risk behaviors, we were unable to show a significant relationship between knowledge of HIV transmission, prevention, or treatment and behavior. However, we found that HIV/AIDS knowledge was modestly but significantly associated with more consistent use of condoms with primary and commercial sex partners, even after controlling for contact with an outreach worker, and that contact with an outreach worker was also an independent predictor of more consistent condom use.

\section{Contact, Knowledge, and Condom Use}

Although methodological differences make comparisons challenging, our analysis found rates of condom use similar to other studies in Vietnam. For example, $36.2 \%$ of respondents in our study reported inconsistent use of condoms with commercial sex clients in last six months, compared to $36-41 \%$ inconsistent use reported in a study of female sex workers in Hanoi [21]. The latter study used a recall period of one month.

Table 6. Multivariable Logistic Regression Analysis of the Effect of HIV-Related Knowledge on Inconsistent Condom Use Among those who had sex in Last Six Months (n=1,983), Unadjusted and Adjusted

\begin{tabular}{|c|c|c|c|c|c|c|}
\hline \multirow{2}{*}{ Outcome } & \multicolumn{2}{|c|}{$\begin{array}{c}\text { Inconsistent Condom Use: } \\
\text { Primary Partner }(\mathbf{n}=\mathbf{1 , 3 9 9})\end{array}$} & \multicolumn{2}{c|}{$\begin{array}{c}\text { Inconsistent Condom Use: } \\
\text { Casual Partner (n=1,981) }\end{array}$} & \multicolumn{2}{c|}{$\begin{array}{c}\text { Inconsistent Condom Use: } \\
\text { Commercial Client (n=1,978) }\end{array}$} \\
\hline Exposures & Unadjusted & Adjusted* & Unadjusted & Adjusted* & Unadjusted & Adjusted* \\
\hline \hline All Knowledge, $\%$ & $0.98(0.97,0.99)$ & $0.98(0.97,0.99)$ & $0.98(0.97,0.99)$ & $0.99(0.98,1.00)$ & $0.97(0.96,0.98)$ & $0.98(0.97,0.99)$ \\
\hline Location & & & & & & \\
\hline An Giang & $0.70(0.48,1.02)$ & $0.67(0.46,0.98)$ & $3.90(2.28,6.65)$ & $3.75(2.19,6.41)$ & $0.70(0.49,0.99)$ & $0.71(0.50,1.02)$ \\
\hline HCMC & $1.09(0.84,1.43)$ & $1.07(0.82,1.41)$ & $1.19(0.92,1.55)$ & $1.16(0.89,1.51)$ & $1.26(0.97,1.63)$ & $1.23(0.94,1.61)$ \\
\hline Hai Phong & $0.92(0.64,1.33)$ & $0.92(0.64,1.01)$ & $2.62(1.63,4.19)$ & $2.59(1.62,4.16)$ & $0.56(0.39,0.80)$ & $0.53(0.36,0.76)$ \\
\hline Hanoi & reference & reference & reference & reference & reference & reference \\
\hline Age & $0.99(0.98,1.01)$ & $0.99(0.98,1.01)$ & $1.01(0.99,1.03)$ & $1.01(0.99,1.03)$ & $0.98(0.97,0.99)$ & $0.99(0.97,1.00)$ \\
\hline Contact $v s$ No Contact* & $0.67(0.53,0.85)$ & $0.72(0.57,0.92)$ & $0.74(0.58,0.94)$ & $0.78(0.61,1.01)$ & $0.59(0.47,0.74)$ & $0.68(0.54,0.86)$ \\
\hline
\end{tabular}

*Each item adjusted for all other variables in table. Confidence interval is indicated in parentheses. HCMC $=$ Ho Chi Minh City 
MARP who reported recent contact with an outreach worker were $22-32 \%$ less likely to report using condoms inconsistently. In other words, they had more consistent condom use. In addition, increased knowledge was associated with more consistent use of condoms with primary and commercial sex partners even after controlling for contact with outreach workers. However, the independent effect of HIV-related knowledge was modest, and the effect of contact on condom use was strongest when considering commercial sex encounters. This may indicate that once knowledge levels are high, outreach programs affect behavior in other ways, such as by transferring skills (how to communicate with partners and negotiate condom use), increasing self-efficacy, interpersonal bonding between peer educators and their clients which may help change social beliefs around condom use, or improving access to condoms in the CSW population. This conclusion is consistent with the findings from other behavior change interventions, which suggest that there are many individual, community, and population level drivers of transmission which influence individual risk behavior, in addition to knowledge $[12,13$, 23]. Future studies should try to measure more intermediaries in the causal pathway from contact with outreach worker to behavior change, to better understand the mechanisms of program impact.

Unlike previous studies, we found that inconsistent condom use was highest with casual partners. Tran, Detels, and Lan (2006) found that inconsistent condom use by female sex workers was lower with irregular (casual) clients, compared to regular clients or primary partners. One possible reason for the difference between our findings and theirs could be that we examined condom use among all MARP and did not limit our analysis to sex workers. We also found that consistent condom use was related to location: respondents in An Giang and Hai Phong were less likely to use condoms consistently with casual partners, compared to respondents in Hanoi. This may indicate that messages from outreach workers regarding sexual behavior in these two provinces are focusing more on commercial sex partners or primary partners, and are not explicitly dealing with the practice of casual sex.

At the time of the study, agencies implementing outreach programs were not yet giving treatment information to clients on a regular basis, which may help explain the consistently low scores among the different MARP groups on treatment knowledge $(41.7 \%$ overall). Knowledge of treatment was highest among IDU, possibly because IDU are more likely to be HIV positive and may have sought out treatment information: of the 376 IDU respondents who reported receiving HIV test results, $47.3 \%$ were positive compared to $6.9 \%$ of non-IDU respondents who were tested.

\section{Contact, Knowledge, and Needle Sharing}

Reported rates of needle sharing were lower in our study relative to previous studies: rates in the four provinces studied ranged from $3.6 \%-26 \%$, compared to $7.6-44.3 \%$ reported by others [24]. Similar to Nguyen et al. (2004), the findings of our study suggest that location is associated with risk behaviors; for example, IDU in HCMC are 2-3 times more likely to have shared needles than IDU in Hanoi. While needle sharing increases the risk of HIV infection, we found that IDU in HCMC were also more likely to clean their needles and other injection equipment before using, compared to IDU in other provinces. Among IDU in HCMC, $62.6 \%$ reported having started/increased cleaning equipment since learning about the HIV/AIDS epidemic, compared to $46.7 \%$ in Hanoi. In places like HCMC, where sharing needles is embedded in the social context of drug use [24], outreach workers may be adapting their messages to emphasize safe cleaning practices for harm reduction.

We found that neither knowledge nor contact with an outreach worker were significantly associated with needle sharing behavior. It is possible that addiction is a stronger determinant of needle sharing than knowledge of ways to prevent HIV infection and the consequences of sharing needles. This suggests that prevention programs may need to invest more in providing services to overcome addiction among IDUs in order to slow transmission rates.

\section{Study Limitations}

A number of considerations bear on the findings of this study. First, because we used a cross-sectional study design, we cannot infer causality. In addition, reported behaviors may have been subject to recall bias. Participants' responses may have been affected by social desirability bias, although the low rates of reported consistent condom use suggest that this was not a major factor.

MARP participants were categorized into groups based on recent contact with an outreach worker, defined as contact within the past six months. Although some participants reported no recent contact with an outreach worker, it is possible that their knowledge and behaviors were influenced by contacts which occurred more than six months ago. This is more likely to have influenced our results for injection drug use, as the IDU population is thought to be relatively stable compared to CSW. As found in other research, the lack of refined exposure measures for classifying participants may have biased results toward the null [18]. In addition, our measure of knowledge included questions meant to cover a broad set of issues and topics related to HIV/AIDS. Participants who did not score high on overall knowledge may still have good understanding of their own personal exposure risk.

\section{CONCLUSION}

Outreach programs are an important component of a comprehensive HIV prevention strategy in Vietnam. This study underscores that these programs can help increase HIV-related knowledge, and influence changes in behaviors to protect against further transmission, particularly regarding more consistent condom use, but that outreach may also affect behavior in other ways such as by increasing skills, self-confidence, motivation, and perceived behavioral control. It is especially important to target additional study and intervention research toward the IDU population in examining the impact of outreach on behavior change. As the HIV/AIDS epidemic in Vietnam matures and increasing numbers of HIV-infected individuals need ART, it will also be critical to improve communication about how and where to access these medications. 


\section{CONFLICT OF INTEREST}

The authors confirm that this article content has no conflict of interest.

\section{ACKNOWLEDGEMENTS}

This study was supported by the Hanoi Mission of the US Agency for International Development (USAID) to the Child and Family Applied Research project at Boston University, Boston, by means of a cooperative agreement (Country Research Activity GHS-A-00-03-00020). The opinions expressed herein are those of the authors and do not necessarily reflect the views of USAID. We acknowledge the study team members Mary Bachman DeSilva, Theodore Hammett, Danielle Lawrence, Kelly McCoy, and Jordan Tuchman for their efforts, and the members of our Vietnamese data collection team and Vietnamese government officials who provided their support and assistance to this project. We are especially grateful to the many individuals in Vietnam who provided information by participating in the surveys.

\section{SUPPLEMENTARY MATERIAL}

Supplementary material is available on the publisher's web site along with the published article.

\section{REFERENCES}

[1] Tran TN, Detels R, Long HT, Van Phung L, Lan HP. HIV infection and risk characteristics among female sex workers in Hanoi, Vietnam. J Acquir Immune Defic Syndr 2005; 39(5): 581-6.

[2] Colby DJ. HIV knowledge and risk factors among men who have sex with men in Ho Chi Minh City, Vietnam. J Acquir Immune Defic Syndr 2003; 32(1): 80-5.

[3] Ministry of Health. HIV/AIDS Estimates and Projections 20052010. Hanoi, Vietnam: Ministry of Health 2005.

[4] World Health Organization, UNAIDS. UNAIDS/WHO epidemiological fact sheets on AIDS and sexually transmitted infections - 2006 Update. Geneva 2006.

[5] Nguyen TV, Khuu NV, Truong PH, Nguyen AP, Truong LX, Detels R. Correlation between HIV and sexual behavior, drug use, trichomoniasis and candidiasis among female sex workers in a Mekong Delta province of Vietnam. AIDS Behav 2009; 13(5): 873-80.

[6] Colby D, Cao NH, Doussantousse S. Men who have sex with men and HIV in Vietnam: a review. AIDS Educ Prev 2004; 16(1): 4554.

[7] Go VF, Frangakis C, Nam le V, et al. High HIV sexual risk behaviors and sexually transmitted disease prevalence among injection drug users in Northern Vietnam: implications for a generalized HIV epidemic. J Acquir Immune Defic Syndr 2006; 42(1): $108-15$.

[8] Ngo DA, Ross MW, Phan H, Ratliff EA, Trinh T, Sherburne L. Male homosexual identities, relationships, and practices among young men who have sex with men in Vietnam: implications for HIV prevention. AIDS Educ Prev 2009; 21(3): 251-65.
[9] Nguyen TA, Nguyen HT, Le GT, Detels R. Prevalence and risk factors associated with HIV infection among men having sex with men in Ho Chi Minh City, Vietnam. AIDS Behav 2008; 12(3): 476-82.

[10] National Institute of Hygiene and Epidemiology. Communitybased HIV, STI, and risk behavior estimates: results from the 2006 integrated biological and behavioral survey (IBBS) in Vietnam. Hanoi: US President's Emergency Plan for AIDS Relief 2007.

[11] Nguyen AT, Nguyen TH, Pham KC, et al. Intravenous drug use among street-based sex workers: a high-risk behavior for HIV transmission. Sex Transm Dis 2004; 31(1): 15-9.

[12] McCoy SI, Kangwende RA, Padian NS. Behavior change interventions to prevent HIV infection among women living in low and middle income countries: a systematic review. AIDS Behav 2010; 14(3): 469-82

[13] Kaponda CP, Norr KF, Crittenden KS, et al. Outcomes of an HIV prevention peer group intervention for rural adults in Malawi. Health Educ Behav 2011;38(2): 159-70.

[14] Morisky DE, Nguyen C, Ang A, Tiglao TV. HIV/AIDS prevention among the male population: results of a peer education program for taxicab and tricycle drivers in the Philippines. Health Educ Behav 2005; 32(1): 57-68.

[15] Kumar MS, Virk HK, Chaudhuri A, Mittal A, Lewis G. A rapid situation and response assessment of the female regular sex partners of male drug users in South Asia: factors associated with condom use during the last sexual intercourse. Int J Drug Policy 2008; 19(2): 148-58.

[16] Needle R, Burrows D, Friedman S, et al. Effectiveness of community-based outreach in preventing HIV/AIDS among injecting drug users. Int J Drug Policy 2005; 2(9): S45-S57.

[17] Sabin L, Hamer D, Vian T, et al. Evaluation of presidential emergency plan for AIDS relief (PEPFAR)-funded community outreach HIV prevention programs in Vietnam: final report. Boston: Boston University Center for International Health and Development 2008.

[18] Morris DS, Rooney MP, Wray RJ, Kreuter MW. Measuring exposure to health messages in community-based intervention studies: a systematic review of current practices. Health Educ Behav 2009; 36(6): 979-98.

[19] Semaan S, Lauby J, Liebman J. Street and network sampling in evaluation studies of HIV risk-reduction interventions. AIDS Rev 2002; 4(4): 213-23.

[20] Ramirez-Valles J, Heckathorn DD, Vazquez R, Diaz RM, Campbell RT. From networks to populations: the development and application of respondent-driven sampling among IDUs and Latino gay men. AIDS Behav 2005; 9(4): 387-402.

[21] Tran TN, Detels R, Lan HP. Condom use and its correlates among female sex workers in Hanoi, Vietnam. AIDS Behav 2006; 10(2): 159-67.

[22] Noar S, Cole C, Carlyle K. Condom use measurement in 56 studies of sexual risk behavior: review and recommendations. Arch Sex Behav 2006; 35(3): 327-45.

[23] Garfein RS, Golub ET, Greenberg AE, et al. A peer-education intervention to reduce injection risk behaviors for HIV and hepatitis $\mathrm{C}$ virus infection in young injection drug users. AIDS 2007; 21(14): 1923-32.

[24] Nguyen TH, Nguyen TL, Trinh QH. HIV/AIDS epidemics in Vietnam: evolution and responses. AIDS Educ Prev 2004; $16(3$ Suppl A): 137-54.

This is an open access article licensed under the terms of the Creative Commons Attribution Non-Commercial License (http://creativecommons.org/licenses/by-nc/ $3.0 /$ ) which permits unrestricted, non-commercial use, distribution and reproduction in any medium, provided the work is properly cited. 